\title{
VEINTICINCO AÑOS DE DERECHOS SOCIALES EN LA EXPERIENCIA CONSTITUCIONAL ESPAÑOLA
}

\author{
MIGUEL ÁNGEL GARCÍA HERRERA \\ Catedrático de Derecho Constitucional \\ Universidad del País Vasco
}




\section{SUMARIO}

1. Los derechos sociales en la lógica del Estado social. 2. DeRechos SociaLES E IGUALDAD SUSTANCIAL. 3. NATURALEZA Y ESTRUCTURA DE LOS DERECHOS SOCIALES. 4. LA REVERSIBILIDAD DE LOS DERECHOS SOCIALES. 5. REGULACIÓNYTENDENCIAS DE LOS DERECHOS SOCIALES. 6. El ESTADO SOCIAL AUTONÓMICO. 7. CONCLUSIONES. 


\title{
VEINTICINCO AÑOS DE DERECHOS SOCIALES EN LA EXPERIENCIA CONSTITUCIONAL ESPAÑOLA
}

POR

\author{
MIGUEL ÁNGEL GARCÍA HERRERA \\ Catedrático de Derecho Constitucional \\ Universidad del País Vasco
}

\section{LOS DERECHOS SOCIALES EN LA LÓGICA DEL ESTADO SOCIAL}

Cuando se quieren identificar las características esenciales del Estado Social que le distinguiría de las formas de Estado precedentes, es opinión doctrinal consolidada señalar a los derechos sociales como uno de los elementos que permite establecer dicha diferencia. En este sentido, la idea de ruptura que acompaña a la aparición de una nueva forma de Estado encuentra su materialización en la regulación de los derechos sociales. Esta afirmación comporta dos exigencias teóricas: por una parte, comprender el significado de la constitucionalización de los derechos sociales; por otra parte, valorar la evolución de los derechos sociales en la crisis del Estado Social y su posible cambio de significado en el proceso de globalización en el que estamos inmersos.

El primer aspecto es relevante porque su tardía elevación al nivel constitucional reclama una explicación. Como es sabido, los contenidos de los derechos sociales surgieron históricamente con anterioridad a la recepción de los derechos de libertad y los derechos políticos que caracterizaron al Estado liberal y su posterior evolución demo- 
crática. Bajo la forma de la beneficencia o de la asistencia social, la preocupación por atender a las necesidades individuales 0 al bienestar colectivo estuvo presente en iniciativas individuales o sociales, y en la acción pública, conscientes de la imperiosa urgencia de atender a los que, por diversas causas, no podían proveerse de los medios indispensables de vida. Tan sólo en momentos puntuales esta preocupación alcanzó un mayor reconocimiento, que se tradujo en la recepción constitucional de fórmulas generales que expresaron una sensibilidad respecto a un estado de cosas insatisfactorio, o en el establecimiento de un sistema de ayudas que persiguió compensar las insuficiencias del mercado. Nos estamos refiriendo, obviamente, a las Constituciones francesas de 1793 y 1848, así como al sistema Speenhamland que recibió una convincente explicación por parte de Karl Polanyi.

El proceso de industrialización transformó las condiciones politico-económicas y provocó el injerto de los derechos democráticos en el Estado liberal. Pero se soslayó la constitucionalización de los derechos sociales. Pero este fracaso fue compensado por la aparición de la normativa laboral que corregía la prepotencia empresarial y por la publicación en Alemania, en los años 80 del siglo XIX, de una legislación social sistemática que instauró un sistema de seguros que se generalizó en Europa.

La constitucionalización de los derechos sociales se produjo en unas condiciones históricas precisas. Este dato se subraya sobre todo por contraste con la experiencia del periodo de entreguerras. A pesar de la incorporación en textos constitucionales como la Constitución de Weimar y la Constitución española de 1931, el intento fracasó. Dos aspectos merecen ser destacados: en primer lugar, en ambos casos las fuerzas políticas y sociales democráticas no fueron capaces de sostener el régimen constitucional instaurado, que pereció por la ofensiva del nazismo alemán y el golpe militar; en segundo lugar, la doctrina alemana mayoritaria demostró su incapacidad para aceptar una concepción normativa de los derechos sociales.

Por ello, la constitucionalización de los derechos sociales no fue una mera ampliación del elenco de los derechos constitucionales sino la expresión de un salto cualitativo que correspondió a una nueva fase del constitucionalismo. Por decirlo brevemente, si antes los derechos sociales expresaban una corrección puntual o mínima de las condiciones de vida, ahora se trataba de insertar los derechos sociales en el modelo constitucional de reproducción social. No se trata, pues, de admitir una evolución natural y espontánea desde los derechos de libertad a los derechos sociales, ni de considerar que se da una sama- 
ritanismo estatal que atiende a las necesidades de quienes carecen de recursos. Antes bien, es la propia estructura del Estado Social la que reclama la regulación constitucional de los derechos sociales.

\section{DERECHOS SOCIALES E IGUALDAD SUSTANCIAL}

Si el Estado Social como principio jurídico sintetiza el conjunto de contenidos que caracteriza a una forma de Estado, los derechos constitucionalizados encuentran su referente cardinal en el principio de igualdad sustancial recogido en el artículo 9.2 CE que, sin perjuicio de las diferencias subrayadas por la doctrina, responde a la inspiración del artículo 3.2 de la Constitución italiana. La referencia a un criterio unificador significa que los derechos sociales no se limitan a satisfacer necesidades sociales e individuales sino que están inscritos en un planteamiento general que les proporciona un fundamento y les dota de un sentido encaminado a la realización del modelo esbozado en la Constitución.

La obligada toma en consideración de la igualdad sustancial impone que realicemos unos breves comentarios sobre esta cuestión. Aunque no ha merecido una gran atención por parte de la doctrina española, se puede coincidir en que estamos simultáneamente ante un concepto tan fundamental como complejo, dificultad que deriva de su carácter evolutivo, que impide aprehender definitivamente el concepto y delimitar sus formas de realización.

La igualdad sustancial, como síntesis de un modelo, incorpora al texto constitucional una tensión permanente e irresoluble. En primer lugar, se da una pugna entre lecturas que apuestan por una interpretación del texto acorde con las exigencias cambiantes, y las que propugnan la tesis de que se reduzca al tenor literal de los preceptos constitucionales. Pero la relación entre los artículos 9.2 y $10.1 \mathrm{CE}$, la conexión entre la igualdad sustancial y el libre desarrollo de la personalidad, imponen una interpretación abierta y dinámica que suponga la permanente apertura hacia nuevos contenidos que realicen los ambiciosos contenidos constitucionales. De ahí que haya autores que apuestan por un planteamiento palengenético que suponga una referencia continua hacia la complejidad y dificultad de realización de los fines constitucionales. La relación entre los preceptos mencionados no puede reducirse inmediatamente a los contenidos constitucionales porque representa la incorporación de una voluntad de evolución capaz de integrar las exigencias que emergen de una transformación 
socioeconómica que adquiere en la actualidad connotaciones inéditas. Sin perjuicio de volver sobre el tema, baste aquí apuntar que el proceso globalizador ha impulsado procesos de emigración de capitales y de inmigración de personas que han modificado los vínculos de solidaridad y transformado el tejido social. Estos cambios no pueden no interpelar en términos novedosos al principio constitucional de la igualdad sustancial y reclamar respuestas idóneas para la materialización del modelo sancionado jurídicamente.

Si consustancial al derecho es cobijar una tensión entre la determinación política del derecho y la realización jurídica de la política, esta contradicción se concentra con especial intensidad en la igualdad sustancial. No yerra quien considera que en torno a ella se solventan algunos de los problemas capitales de la colectividad. En el fondo, significa convertirla en medio y fin de un proceso inacabado, puesto que el nivel de igualdad que se alcanza representa la materialización de los procesos democráticos y la expresión de la virtualidad de la dotación constitucional de los derechos y libertades. Y al mismo tiempo, es punto de arranque para nuevos logros que posibiliten la generalización en cada fase histórica de la consecución del libre desarrollo de la personalidad. La igualdad sustancial es el precipitado en el que convergen el reconocimiento de derechos $y$ sus condiciones materiales de realización libre y efectiva, la atribución de titularidades jurídicas y su efectiva plenitud, la convivencia entre derechos económicos y derechos sociales, de libertades y redistribución, de autonomía privada y solidaridad, tanto pública como privada. Frente a la denuncia del riesgo de una democracia sin demócratas, se trata de alcanzar una democracia de calidad que propone una definición de la comunidad política que favorezca la creación de vínculos sociales, sólo posibles desde la disposición de condiciones materiales capaces de resistir un análisis desde el prisma de la dignidad de la persona.

A pesar de las dificultades lógicas y gramaticales de la igualdad sustancial, es insoslayable enunciar indicaciones que permitan captar su contenido. Nuestra Constitución ha sido generosa en su configuración al referirse simultáneamente a la promoción de condiciones, la remoción de obstáculos y a facilitar la participación. De este enunciado podemos extraer algunas apreciaciones que nos permitirán su comprensión. En primer lugar, no se trata de un planeamiento definitivo o reactivo con el que evitar el deterioro de la igualdad, sino de una propuesta activa de construcción de condiciones materiales y personales susceptibles de realizar los fines proclamados. En segundo lugar, debemos recordar la tesis de Ferrajoli ya enunciada hace años: tanto la igualdad formal como la sustancial no son descripciones sino juicios 
de valor basados en la diversidad entre los hombres. Pero mientras que con la igualdad formal se considera que los hombres deben ser considerados como iguales a pesar de que son distintos y prescindir de sus diferencias raciales, sexuales, religiosas, etc., con la igualdad sustancial se postula que los seres humanos deben ser hechos tan iguales como sea posible y tomar en cuenta, precisamente, que son desiguales en el ámbito social y económico. Ello significa que en el primer supuesto se respetan y se protegen las diferencias, y que en el segundo caso se reacciona frente a ellas para su reducción o eliminación. Este planteamiento es una buena plataforma para comprender el alcance de la igualdad sustancial. Frente a las tesis tan en boga que pretenden reducir su significado a la igualdad de oportunidades y desentenderse de los resultados, la interpretación más ajustada es la que elabora una visión conjunta que atiende a los medios dispuestos $y$ a los logros alcanzados.

Si seguimos la sugerencia de Rescigno de tomarnos en serio el principio de igualdad sustancial, debemos convenir que su fundamento reside en la aceptación de la divergencia entre el modelo constitucional y la realidad de los derechos y libertades, entre la distancia entre un reconocimiento forma de atribuciones jurídicas y en la difícil realización de sus posibilidades. Hay, pues, una imposibilidad de hecho de realizar lo que se ha reconocido jurídicamente, y ello por motivos que son contrarios a la propia Constitución, porque son limitaciones o negaciones que carecen de fundamento.

La aceptación de esta realidad criticable invita a proceder a la clasificación de los supuestos que inciden en la irrealización del modelo constitucional. Si persevera la desigualdad es debido a que no hay condiciones prácticas para el ejercicio de la libertad o la consecución de la igualdad, o bien porque son escasos o inexistentes los derechos reconocidos. Son dos planos distintos pero que están intrínsecamente conectados, pues la ausencia de uno de ellos genera la reaparición del dedo acusador del artículo 9.2 que reitera su denuncia. No basta, una vez más, con el reconocimiento del derecho si, finalmente no se dispone de las condiciones para su efectivo ejercicio. Dada la indeterminación jurídica de los contenidos de los derechos, el legislador es más proclive al reconocimiento del derecho que a incidir sobre las condiciones materiales. Es más fácil reconocer el derecho a la salud que asignar los recursos necesarios para que la curación sea una realidad al alcance de los miembros de la sociedad, y no sólo de los más pudientes. De ahí que no todo consista en la previsión de derechos individuales sino que la adopción de medidas administrativas y financieras es el complemento indispensable, porque aquéllas son los ins- 
trumentos para actuar las previsiones constitucionales. Paradigmático en este sentido es el derecho a la enseñanza como condición básica para que los niños y jóvenes adquieran la madurez que les convierta en efectivos ciudadanos. Resulta imprescindible la dotación de medios para que la formación que capacita para la asunción de una función en el conjunto social sea real, y para que cada uno encuentre los cauces que le permitan aprovechar las condiciones intelectuales. Pero, a su vez, la escuela y la universidad necesitan que existan unas condiciones de familia, vivienda, alimentación, etc. Sin ellas la enseñanza topa con un muro insuperable y perpetúa crónicamente las desigualdades de hecho.

Por último, hay que referirse a la participación de todos los ciudadanos en la vida política, económica, cultural y social. Con la idea de participación se alude a un modelo en el que conjuntamente la ciudadanía forma parte y toma parte, está integrada en el colectivo referente de la decisión a cuya determinación concurre. La amplitud de ámbitos sobre los que se aplica permite deducir que ni se identifica con el espacio político, ni se limita sólo al aspecto procedimental que comporta la delegación, sino que tienen un alcance que supera la óptica formal para incluir la obligada contemplación de la democracia sustancial, entendida como control social ejercido democráticamente sobre los elementos basilares de la colectividad. Además, la referencia genérica a los ciudadanos que operan en las distintas esferas de la sociedad supone su comprensión en el papel que adoptan en dichos ámbitos. De esta manera, hay que admitir que implícitamente la Constitución asume que la participación en la vida económica la realiza el ciudadano en su condición de trabajador, lo que comporta su protagonismo en el conflicto en el proceso productivo, asi como en la distribución. Sólo de esta forma es posible concebir la realización del modelo constitucional al que debe, no obstante, actuarse en el marco complejo de la definición de una regulación económica que recoge derechos y libertades. No se trata de olvidar las coordenadas de la reproducción social, pero tampoco que sus consecuencias negativas exigen la corrección por medio de un conflicto ordenado que permite la convivencia de intereses enfrentados. Es, pues, una idea de democracia no limitada ni en las formas ni en los contendidos, ni en los sectores de aplicación, y con una vocación histórica de realización ajustada a las cambiantes condiciones.

Además se da una relación entre medios y fines. Se trata de conseguir la participación, pero ésta, a su vez, sólo será conseguible gracias a la previa participación. El avance que supone lograr la efectividad de los derechos se inscribe en un contexto de convivencia y por 
ello su finalidad consiste en la integración en la comunidad alcanzable por medio del "ser parte". Pero esta integración no es el fruto de una evolución espontánea sino el resultado de la presión ejercida por los sujetos débiles para lograr la plenitud de sus derechos que, en nuestra Constitución, significa incidir en la producción y distribución de la riqueza y la aplicación de recursos para superar las situaciones de desigualdad. En consecuencia, la participación engendra participación para lograr el objetivo final del desarrollo de la persona.

Ciertamente, la igualdad sustancial plantea muchas cuestiones acerca de los sujetos activos $y$ pasivos, de los contenidos y materias, de las formas de realización que no pueden abordarse. $Y$ es en esta complejidad lógica y gramatical la que explica que se opte por su uso complementario o innecesario en una argumentación ya acabada y basada en otras consideraciones, o su postergación en beneficio de la aplicación razonable de la igualdad formal. Sobre este tema volveremos más adelante.

Sin embargo, ya se ha superado la consideración programática de las normas constitucionales y se admite sin reservas la efectividad de cada uno de los preceptos. En este planteamiento la regulación de la igualdad sustancial adquiere una especial relevancia por su capacidad de proporcionar una visión unitaria de la orientación que debe adoptar la evolución social a la luz de las prescripciones sociales. Supone disponer permanentemente de la versión que da la Constitución de su realización, que se traduce en la superación de las desigualdades personales $y$ estructurales en el marco de compatibilidad del conjunto de valores $y$ derechos. La opción básica significa que la labor del legislador y la interpretación constitucional se realizan en pro de la consecución de un modelo de emancipación que se define históricamente y está abierto a la integración de nuevas necesidades y a la superación de los obstáculos socioeconómicos perturbadores de la plenitud de los derechos. Si la dificultad de su utilización disuade o ahuyenta su aplicación, estaremos ante un incumplimiento de la Constitución y ante la dimisión del jurista de su tarea de explorar los límites y atisbar las potencialidades y modulaciones en cada momento de este precepto de largo alcance. Tarea que es decisiva en esta ocasión, puesto que la apertura de la norma empuja a explorar las posibilidades en todos los sectores y a demostrar la sensibilidad ante nuevas desigualdades y obstáculos. Ejemplos de dificultades y desafíos los hay en la actualidad debido no sólo al desarrollo de la conciencia social que reacciona ante hechos antes tolerados, sino también ante nuevos supuestos que se manifiestan en el proceso de globalización concretados en la exclusión, marginalidad y emigración, fenómenos todos 
ellos ante los que el imperativo de la igualdad sustancial no puede mostrarse indiferente.

La comprensión de la igualdad sustancial requiere establecer una compleja relación con los contenidos constitucionales, para así comprender el alcance de su aportación. Además de que se nos presenta, como veremos más adelante, como substrato de libertad y de la democracia, es preciso en este momento de la exposición insistir en las relaciones con la igualdad formal.

Ha sido habitual en la jurisprudencia constitucional establecer la conexión entre el artículo 9.2 y $14 \mathrm{CE}$. Básicamente la igualdad sustancial proporciona un apoyo superfluo o un refuerzo al artículo 14, de forma que si se prescindiera de la referencia al artículo 9.2 la argumentación no sufriría y se alcanzaría el mismo resultado. Sin embargo, la relación establecida es discutible, pues reducir la igualdad sustancial a mero complemento de la igualdad formal supone reproducir la tesis doctrinal de la igualdad sustancial como simple especificación de la igualdad formal y renunciar a valorar la novedad que supone el contenido del artículo 9.2. Como ha sido expuesto, la igualdad sustancial no puede identificarse con la igualdad formal ni con su actualización, que demanda el trato desigual para lo que es desigual. La igualdad sustancial no consiste sólo en la toma en consideración de las diferencias fácticas porque su formulación en el artículo 9.2 realiza una aportación de valores y finalidades que desbordan las previsiones del artículo 14. Como este precepto se limita al trato igual y a sus matizaciones ante la diversidad, no es posible desde él, pero sí desde el artículo 9.2, fundamentar las intervenciones materiales que corrijan los obstáculos y favorezcan la participación. Por tanto, no cabe integrar la igualdad sustancial en la igualdad formal porque se cercena el texto constitucional y se liquida uno de los contenidos innovadores incluidos en la Constitución de 1978.

\section{NATURALEZAY ESTRUCTURA DE LOS DERECHOS SOCIALES}

A pesar de su reconocimiento constitucional, los derechos sociales son objeto de debate precisamente por su naturaleza. Aunque su dicción literal es inequívoca, numerosos autores continúan indicando la conexión entre reconocimiento y realización por medios materiales como justificación de una escisión entre el enunciado y la eficacia jurídica del derecho y como explicación de las dudas sobre la considera- 
ción de estos derechos como ius cogens. Las numerosas aportaciones doctrinales y jurisprudenciales que consideran inadmisible la escisión entre el reconocimiento del derecho y su protección jurídica no sirven para acallar las posiciones de quienes prosiguen diferenciando entre derechos de primera segunda y segunda generación, consideran primordiales los primeros e, incluso, admiten, en la actual situación de revisión, la posibilidad de su eliminación constitucional. De esta posición crítica no están alejados los que detectan una contradicción entre derechos sociales y democracia por considerar que la exigencia de realización de los primeros comporta limitar el margen de decisión de las mayorías, que se ven constreñidas por la exigencia de eficacia jurídica de unas pretensiones que son costosas económicamente.

La pretendida contraposición entre derechos políticos y sociales se ha superado para admitir la sustancial semejanza entre ellos.

Los derechos sociales han sido objeto de una ofensiva planteada en muy diversos frentes que han cuestionado su terminología, lógica, estructura y eficacia. Precisamente uno de los aspectos destacables es que ha soportado esta aguda polémica y que el resultado ha sido la plena afirmación de los derechos sociales y el reconocimiento de su estatus.

Uno de los flancos más enconados ha consistido en la contraposición entre derechos de libertad y derechos sociales y la pretendida superioridad de los primeros. Es incontrovertible la relevancia que históricamente han alcanzado los derechos de libertad, convertidos en el objeto central del derecho constitucional y en la fuente de legitimidad del poder. Desde una cierta comprensión de la persona, la libertad se convierte en el perno de las relaciones sociales y políticas. Al afirmar el conflicto individuo-poder, la Constitución traduce la desconfianza ante el poder en un conjunto de límite de las instituciones y de una técnica de la libertad por medio de la preservación de la autonomía y de la compatibilidad de libertades. No resulta sorprendente que de esta entronización de la libertad se derive su antagonismo con la igualdad, puesto que ésta es comprendida como un factor que demanda una intervención pública correctora que, por definición, contrasta con la exigencia de decisión sin interferencia propia de la libertad y garantía de la esfera de autonomia personal.

Cierto es que análisis más rigurosos han disuelto el pretendido antagonismo y que en los orígenes del régimen liberal se ha verificado el aprecio por los derechos sociales como complemento indispensable de la libertad. Pero en todo caso, el hecho histórico es que la realidad social de la ayuda pública no logró estatus jurídico y la construc- 
ción del nuevo Estado se realizó sobre unos derechos considerados consenso básico e imprescindible del orden social liberal.

Pero este desfase histórico se ha superado gracias a que se ha desmontado la pretendida contradicción lógica entre derechos de libertad y derechos sociales. La superación de las condiciones sociales que permitieron la exclusiva y reducida valoración de la libertad impuso una revisión de las relaciones con los otros derechos. La libertad requiere unas condiciones materiales sin las que para ciertos sectores sociales el reconocimiento de la libertad es nominalismo jurídico. Por otra parte, la libertad se realiza políticamente como democracia que sólo es posible desde la igualdad de todos los integrantes de la colectividad. Se establece, pues, una relación inescindible entre los tres elementos: libertad, participación e igualdad, que se reclaman, se influyen y se desarrollan conjuntamente. Sin el desarrollo simultáneo de los tres contenidos se produce una perversión que reduce la libertad a elitismo, la democracia a procedimiento formal y la igualdad a declaración retórica.

La revalorización lógica de los derechos sociales y la aceptación de su compatibilidad con los principios constitucionales y de su relación interactiva con los derechos de libertad, ha permitido realizar una revisión de la estructura de los derechos sociales y verificar el supuesto antagonismo entre los derechos sociales y los derechos de libertad.

Es clásica la contraposición entre los derechos de libertad que se oponen, que imponen su abstención y preservan su autonomía. Por el contrario, con los derechos sociales se contempla la prestación de los poderes públicos, dependiendo de la regulación normativa y de la disposición de medios materiales, personales y financieros. De ahí que, en síntesis, se recurra a la dualidad entre unos derechos contra el Estado $y$ otros que se presentan ante el Estado.

A estas alturas es un lugar común considerar insuficiente esta polarización. Cuando se procede a diseccionar los elementos estructurales de ambos grupos de derechos se concluye en la imposibilidad de mantener la agrupación tradicional. La idea de unos derechos de libertad asociada a la abstención del Estado como si la actividad pública fuera suficiente para la efectividad del derecho, es insostenible. No causa sorpresa afirmar que los derechos de libertad también requieren medios económicos y materiales, y que sólo su aplicación garantiza que el reconocimiento del derecho tenga su traducción práctica. Cualquier derecho de libertad requiere, en última instancia, una tutela judicial que sólo es posible por medio de la disposición por parte del Estado de una organización costosa económicamente para la satisfacción del dictado constitucional. 
De la misma forma que se ha superado la visión simplificada de los derechos de libertad para complementarlos con la referencia a la aportación del Estado, también se ha tenido que corregir la comprensión unidimensional de los derechos sociales. Esta nueva lectura reclama contemplar su complejidad y referirse a más aspectos que la consideración del contenido prestacional. La idea más amplia de los derechos sociales se alcanza cuando se comprende que son capaces de albergar los diversos contenidos que configuran la estructura compleja de los derechos fundamentales. Efectivamente, si reconocemos que se pueden agrupar los derechos fundamentales en cuatro apartados que traducen las situaciones jurídicas de los titulares, la bondad de esta clasificación se demostrará en la posibilidad de integrar a cada uno de dichos derechos en los distintos apartados. La novedad ha consistido en que se ha superado la identificación de cada derecho con un único grupo para admitir que un derecho constitucional puede incorporar en su contenido diversos aspectos que nos dan una visión más amplia de la complejidad del derecho. Esto no significa que la convivencia de diversos contenidos no permita su graduación y el reconocimiento de uno de ellos como calificación primordial de la identidad del derecho. Un ejemplo recurrente de esta lectura omnicomprensiva es el derecho a la salud, derecho prestacional por el que se reclama la disposición de medios sanitarios y hospitalarios para la prevención y recuperación de la salud, pero que contiene también elementos de participación y autonomía por medio del consentimiento informado o el rechazo a la acción terapéutica. De esta forma se vuelve a superar la contraposición entre los derechos de libertad y los derechos sociales puesto que no son susceptibles de encajonamiento rígido en una clasificación o en el reconocimiento de contenidos incompatibles entre las categorías de derechos.

Esta aceptación del contenido heterogéneo de los derechos sociales lleva a la conclusión de la imposibilidad de admitir una categoría unitaria. Esta cuestión, que ha absorbido mucha energía doctrinal en el intento de establecer la identidad de los derechos sociales, hoy en dia queda superada al reconocer que en los distintos derechos se pueden reconocer distintas situaciones jurídicas que no son reconducibles a unidad puesto que, como hemos dicho, conviven posibilidades diversas que mantienen relaciones específicas entre sí.

Como ha puesto de relieve Luciani, también se ha considerado inservible la conexión entre derechos sociales y poderes públicos, como rasgo identificador. La normal participación y protagonismo institucional en la prestación de los derechos sociales no debe desembocar, como más tarde veremos, en la consideración de que sólo las 
Administraciones Públicas están habilitadas para atender las necesidades individuales y sociales. En la realización de los derechos sociales, lo relevante es la satisfacción real de las expectativas con independencia del sujeto o cauce de la prestación. Una vez más se vuelve a superar otra polaridad que se consideraba distinguía a cada categoría de derecho, aspecto que, por otra parte, está sometido en la actualidad a una profunda revisión.

Contemporáneamente a la relectura de la estructura del derecho, se ha procedido a la interpretación crítica de la naturaleza de los derechos sociales. Mucho se ha evolucionado en la comprensión de los derechos sociales. Las reconstrucciones doctrinales han puesto de relieve las diversas lecturas que se fueron sucediendo desde que se produjo la constitucionalización de los derechos sociales. Las primeras formulaciones, que explicitaban la profunda desconfianza, se pronunciaban a favor de la naturaleza programática y su consiguiente desvalorización al quedar reducida a objetivo a conseguir según los criterios discrecionales del poder. La negación del carácter jurídico realizada en el período de entreguerras por prestigiosos juristas alemanes era incompatible simultáneamente con la naturaleza de la Constitución y con la relevancia de los derechos sociales en la estructura del Estado. Bastó que se considerara a la Constitución como unidad y como norma jurídica para que fuera insostenible la aceptación de un doble nivel normativo en el seno de la Constitución. La necesidad de aceptar la visión unitaria de la Constitución condujo al reconocimiento del valor jurídico de todos los preceptos y a la consiguiente aplicación de tal concepción también a los derechos sociales. Este primer paso fue seguido por otras interpretaciones que apuntaban a la aplicación a los derechos sociales de la teoría administrativa de los intereses. El siguiente paso consistió en la aceptación no sólo del carácter jurídico de los derechos sociales sino también de su naturaleza de derechos. Esta conclusión cerraba algunas cuestiones y abria nuevos interrogantes. Clausuraba un debate especialmente sensible puesto que al depender para su realización de los elementos personales y de los fondos presupuestarios se cuestionaba la obligatoriedad constitucional de los derechos sociales, puesto que su futuro dependía de la voluntad política de los parlamentos y del desarrollo de la lucha política y del conflicto social. Por otra parte, se iniciaba una nueva reflexión que pretendia pasar de la normatividad a la prescriptividad y se reflexionaba sobre la naturaleza constitucional de los derechos sociales, aspecto acentuado en nuestra Constitución por su particular orden interno que distribuye los derechos en dos capítulos que hay interpretar a la luz del artículo 53. 
En estas condiciones no sorprende que se haya pretendido excluir a los derechos sociales del conjunto de los derechos fundamentales. Diversos argumentos se oponen a este planteamiento. En primer lugar, de tipo histórico porque supone reservar la calificación de fundamentales al conjunto de derechos definido en el momento de la construcción del Estado liberal, que respondía a una determinada concepción de la relación poder-individuo y que se ajustaban a los intereses de la burguesía. Pero la evolución del constitucionalismo se ha realizado precisamente con la incorporación de nuevos derechos que han traducido con la misma intensidad las nuevas necesidades $y$ expectativa sociales en un proceso no concluido, como se comprueba en la actualidad con los problemas planteados en el campo de la biotecnología. En segundo lugar, desde un punto de vista sistemático, es más acorde con la comprensión de nuestra Constitución optar por la interpretación que permite la máxima eficacia de un derecho que sólo responde al dictado constitucional de la realización de la personalidad del individuo. En tercer lugar, la consideración de los derechos sociales como derechos fundamentales derivaría no sólo de la necesidad de una visión de conjunto de los derechos sociales y de libertad, sino de la protección que deben recibir para asegurar los niveles básicos de existencia y dignidad humanas, sólo concebibles en la complementariedad entre ellos. Frente a ello, no cabe objetar la diferente protección jurídica dispensada. Que los derechos de libertad tengan protección desde la misma Constitución, no debe conducir a menospreciar la protección de los derechos del Capítulo III del Título I frente al legislador. $Y$ en todo caso, ambos derechos ambos tipos de derechos tienen protegido el contenido constitucional sin que la mención al contenido esencial referido a los derechos del Capítulo II proporcione cobertura para no reconocer que los derechos del Capítulo III también tienen protegido el contenido mínimo del derecho. La protección jurídica de dicho contenido viene dispensada por la incorporación en la Constitución, sin que sean significativas a estos efectos las referencias del artículo 53.2 CE.

Para reforzar la naturalezà de los derechos sociales y su identificación como derechos fundamentales, podemos acudir, por último, a la distinción de Ferrajoli entre los derechos y sus garantías. La denuncia de ausencia de garantías de los derechos sociales que los diferenciaría de los derechos fundamentales, significa asumir una confusión que lleva a negar la existencia del derecho cuando falta la garantía. Pero para el autor italiano la existencia del derecho se deriva de su reconocimiento normativo y no de la precisión de su garantía, que debe estar establecida por el legislador. Que los derechos sociales no 
dispongan de garantías positivas parangonables a las previstas en los derechos de libertad, no es causa para negar su naturaleza de derechos equiparables a las del resto de los derechos. Conviene, además, no confundir e identificar la realización política con la posible realización técnica de las garantías. Aunque históricamente han dependido de la discrecionalidad administrativa, nada impide, y en esta línea se ha avanzado, que se diera una construcción de los derechos sociales basada en los criterios de gratuidad, obligatoriedad y universalidad y en el establecimiento de obligaciones legales que no dejaran espacio para la autónoma gestión administrativa de las prestaciones sociales. En expresiva frase, se trata de evitar que la concepción del derecho se reduzca a la realidad histórica del derecho.

\section{LA REVERSIBILIDAD DE LOS DERECHOS SOCIALES}

Si bien no se puede negar la posibilidad de que la regulación ordinaria proceda a recortar las facultades reconocidas en los derechos o reduzca el ámbito de autonomía de las libertades, sin embargo, esta cuestión acompaña inexorablemente la reflexión sistemática sobre los derechos sociales, que son analizados desde la perspectiva de su irreversibilidad. Aunque en la actualidad los derechos de libertad y los derechos sociales gozan de la misma relevancia axiológica, y no debería darse este distinto trato teórico, sobre todo si recordamos que los derechos sociales son básicamente la posibilidad de los derechos de libertad, resulta inevitable afrontar el problema de la reversibilidad de los derechos sociales.

Como es sabido, con dicho término se suscita el tema de la posible reducción o no del nivel prestacional establecido en la normativa de desarrollo constitucional. Admitir la irreversibilidad supone asentar una dinámica de crecimiento ilimitado que sólo consiente una fase meseta y un nivel de estabilización, como paso previo para un incremento ulterior del contenido prestacional. Aceptar la reversibilidad significa que lo hoy concedido puede ser mañana recortado y que los derechos sociales están sometidos a un flujo y reflujo continuo, de forma que el nivel prestacional alcanzado no está garantizado, sino que está sometido a las valoraciones políticas y económicas de las sucesivas mayorías.

No son pocos los argumentos que se pueden esgrimir a favor de la irreversibilidad. De acuerdo con las premisas sentadas anteriormente, si los derechos sociales se inscriben en un programa de reali- 
zación de la igualdad sustancial, que persigue en un contexto de producción capitalista superar las desigualdades, es razonable pensar que el desarrollo constitucional ha permitido avanzar en dicho sentido y que es sólo una fase más en el proyecto inacabado de realización de la igualdad sustancial. Retroceder respecto a lo alcanzado supondría añadir un alejamiento adicional respecto al modelo normativo ideal. Además entra en contradicción con la legitimación democrática del poder, porque en la constitución del Estado Social el poder se basa no sólo en procedimientos sino en la eficacia de la solución de las necesidades sociales. Como ya se apuntó, en la lógica del Estado Social, el consenso se basa en la relación entre la garantía del modelo económico y las directrices de redistribución de la riqueza en una evolución emancipadora de largo alcance.

Mucho se ha debatido sobre esta cuestión, porque se ha considerado auténtico banco de prueba de la viabilidad y verosimilitud de la regulación del Estado Social. Para algunos la cláusula del Estado Social proporciona una garantía institucional del nivel prestacional alcanzado; otros estiman que es admisible la omisión constitucional pero que una vez que se procede a la actuación constitucional no cabe la negación sino sólo la reforma. Se adentra esta posición teórica en una intrincada maraña de términos imprecisos, puesto que obliga a trabajar con contenidos básicos, con condiciones económicas, con relaciones con otros derechos, con el margen de apreciación del legislador democrático, con la consolidación del desarrollo constitucional. Como primera afirmación, sería conveniente evitar tanto las posiciones extremas que postulan desarrollos unidireccionales de avance ilimitado, como las tesis que consagran lo posible, eufemismo económico-financiero, como parámetro fundamental de comprensión de la actuación de los derechos sociales.

Aunque nuestro Tribunal Constitucional tiende a hurtar el debate, camuflarse detrás de la igualdad formal y apelar a la autocontención en aras del respeto al legislador, ha realizado algunas afirmaciones que, en esta selección mínima, permiten observar la dificultad $y$ la contradicción que acompañan a estos temas. En primer lugar hay que recordar la aislada y lejana opinión de la STC 81/1982 sobre el amparo que presta la Constitución a las conquistas sociales ya conseguidas: "Dado el carácter social y democrático del Estado de Derecho que nuestra Constitución erige y la obligación que al Estado imponen los arts. 9.2 y 35 de la Constitución de promover las condiciones para que la igualdad de los individuos y los grupos sean reales y efectivas y la promoción a través del trabajo, sin que en ningún caso pueda hacerse discriminación por razón del sexo, debe entenderse que no 
se puede privar al trabajador sin razón suficiente para ello de las conquistas sociales ya conseguidas." Por otra parte, la STC 134/1987 excluye la aplicación de la irregresividad a nuestra Constitución. Evitando toda disgresión abstracta, considera que los artículos 41 y 50 no avalan su integración en nuestro ordenamiento, puesto que no se puede sostener que la Constitución obligue a que se mantengan todas y cada una de las pensiones iniciales en su cuantía prevista ni que todas y cada una de las ya causadas experimenten un incremento anual. Especialmente en relación con las pensiones, el Tribunal Constitucional recuerda que no puede prescindirse de las condiciones económicas de cada momento y de que los recursos son escasos para atender a las necesidades sociales. Finalmente, como en el caso concreto se está analizando una pensión que se considera alta económicamente, elTribunal concluye que las declaraciones constitucionales pueden ser invocadas para actualizar las pensiones bajas y limitar las altas teniendo en cuenta la limitación de los recursos.

A pesar de su pronunciamiento de la STC 81/1982, el Tribunal Constitucional se encuentra en la STC 134/1987 con un supuesto favorable para realizar una comparación entre pensiones altas y bajas y realizar un pronunciamiento favorable a la congelación de las pensiones altas. Pero los Magistrados constitucionales, al concentrarse en el contraste en pensiones altas-bajas, desaprovecharon la oportunidad de analizar con más detenimiento esta compleja cuestión. En todo caso, estos dos posicionamientos extremos nos delimitan el campo de juego en el que encontrar posiciones más matizadas y acordes con el texto constitucional.

Son muchos los argumentos que pueden esgrimirse en favor de la irreversibilidad de los derechos sociales, tanto de índole general como ajustados a la regulación específica de cada uno de ellos. Es tanta la distancia entre la realidad y el modelo normativo que cualquier avance todavía queda lejano del proyecto constitucional: más aún si además se retrocede. Pero el curso de los acontecimientos confirma que se impone la propuesta reductora del acceso y contenido de las prestaciones en aras de unas exigencias financieras que están condicionadas por datos económicos más próximos a la primacia del mercado que a la realización del modelo del Estado Social.

Instalados en la óptica de la revisión de los logros alcanzados, el problema se desplaza hacia la existencia de límites a la degradación de los derechos sociales: o dicho con otras palabras, si el legislador democrático puede traducir cualquier política económica apoyada electoralmente. Es claro que siempre está el tope constitucional que impi- 
de la negación flagrante del contenido de los preceptos constitucionales. Pero el problema no consiste en la contraposición ConstituciónLey que es insostenible en el actual modelo normativo, porque la cuestión es si se pueden establecer límites con unos determinados datos normativos.

En principio, hay que reiterar que el texto constitucional es una compleja trama de valores, derechos, intereses que se resiste a lecturas unidimensionales. Sólo en la visión que dé cuenta de esta trabazón forjada en el consenso constitucional, puede encontrarse el criterio que permita atender a la complicada y obligada convivencia de contenidos contrapuestos. Cierto es que la Constitución ha querido que los derechos sociales se precisen por medio de una legislación que gradualmente procede a su desarrollo o a través de regulaciones sectoriales que contemplan aspectos parciales. Pero esta actuación no invierte los términos de la relación entre normas. Los derechos sociales constitucionales sólo pueden ser regulados y precisados por medio de leyes que encuentran su fundamento en su adecuación a la Constitución. Para toda revisión de contenidos de los derechos sociales la Constitución establece el límite en función del concepto constitucional de dichos derechos, que no sólo no pueden ser negados o desconocidos, sino que también tienen que ser respetados en su contenido constitucional. Este contenido, a su vez, se define históricamente. Cada fase de desarrollo determina el significado de los niveles de vida social acordes con la dignidad de la persona, que tienen que ser atendidos por la intervención del legislador ajustada a los cambiantes parámetros de una Constitución que se hace en el proceso de su desarrollo.

Además, la complejidad de contenidos de la Constitución obliga a realizar la operación de ponderación de los derechos e intereses constitucionalizados. Se trata de volver a operar con los elementos que acompañan a los derechos sociales: la realización legislativa pero no la ilimitada discrecionalidad del legislador. La conexión de los derechos sociales con los medios materiales no puede justificar su subordinación a éstos desconociendo su relieve constitucional. La ponderación no puede quedar a expensas de la mera voluntad sin tomar en consideración el equilibrio que debe producirse entre los distintos contenidos constitucionales. No debería ser suficiente la mera referencia a problemas económicos sin contemplar simultáneamente el conjunto de decisiones adoptadas, no sea que los desequilibrios financieros puedan provenir de decisiones adoptadas en detrimento del modelo constitucional. 
Cierto es que los términos utilizados adolecen de indeterminación. Pero es una ambigüedad no inferior a la existente en otros campos constitucionales. No puede negarse, no obstante, que en esta ocasión se da una dificultad adicional al intentar conciliar la normatividad y la realización de los derechos, el respeto a la mayoría parlamentaria y el papel de defensa del modelo constitucional que compete al Tribunal Constitucional, que debe recurrir a aquellas decisiones que favorezcan la convivencia de los derechos individuales y de la igualdad sustancial. Será a la luz de estas perspectivas cuando el tema de la regresividad de los derechos sociales se podrá afrontar adecuadamente.

\section{REGULACIÓNYTENDENCIAS DE LOS DERECHOS SOCIALES}

Expuestas algunas consideraciones generales sobre la teoría de los derechos sociales, procedemos a continuación a revisar brevemente su desarrollo normativo y los cambios incorporados en su régimen jurídico.

Aunque uno de los temas doctrinales más obsesivos ha sido el de la efectividad jurídica constitucional, y el consiguiente riesgo de la ineficacia de unos derechos sociales entregados a la voluntad política del Poder Legislativo, esta cuestión ha quedado en gran medida superada en la evolución del régimen constitucional español porque se ha procedido a una regulación legislativa que ha atendido formalmente las previsiones constitucionales.

Se abre aquí una importante cuestión que debemos soslayar para limitarnos a unas breves consideraciones. Ha sido opinión común la debilidad e insuficiencias del sistema prestacional recibido del régimen franquista. La carencia de libertades impedía la defensa de los intereses de los trabajadores y sectores sociales desfavorecidos. Hasta el advenimiento de la democracia las autoridades públicas se guiaban por la doble consideración de una ideología asistencial de inspiración religiosa que se traducía en un sistema de ayuda a los pobres y marginados, en la respuesta a las demandas que provenian del proceso de industrialización y a la atención de ciertas necesidades sociales para compensar la ausencia de libertades. En este contexto se procedió a establecer un sistema de seguridad social, a favorecer el acceso a la enseñanza para responder a las necesidades tecnológicas, a disponer ayudas a la vivienda, etc., sin embargo, la imposibilidad de 
defensa política de los sectores afectados acarreaba la primacía de una concepción paternalista lastrada además por la escasez de recursos económicos. Esta compleja situación, caracterizada por la inexistencia de un sistema de bienestar y por las expectativas y demandas de la población, fue la difícil herencia que recibió el régimen democrático, cuya primera respuesta consistió en el consenso que se alcanza sobre ciertos contenidos sociales en los Pactos de la Moncloa y en disponer un articulado sistema constitucional de derechos sociales coherentes con los postulados ideológicos y las bases políticas y económicas del Estado Social que abarcaba la existencia de los ciudadanos, desde la cuna, con la protección de la familia, a la tumba, con la ayuda a la tercera edad. De todas formas, el retraso acumulado de décadas, en comparación con las experiencias socialdemócratas europeas, supondrá una rémora considerable que no conseguirá paliar suficientemente el esfuerzo que se realizará en las dos décadas finales del siglo XX.

Ocupados los primeros años en la consolidación del nuevo orden democrático, la década de los ochenta será el marco temporal en el que se proceda a poner los cimientos del modelo de bienestar español. Prescindimos en esta ocasión de la caracterización general del modelo y de su aproximación a las categorías elaboradas por la doctrina, para centrarnos exclusivamente en el desarrollo normativo.

Se coincide en reconocer el esfuerzo de universalización que se ha realizado. La legislación de desarrollo ha transformado las previsiones constitucionales en derechos que han dotado de efectividad a los derechos sociales de la Constitución de 1978. Así, podemos recordar la legislación educativa de los diversos niveles de enseñanza que garantizan el acceso a la educación, la Ley 14/1986 de sanidad y el Real Decreto 1088/1989 que han universalizado la atención sanitaria, la Ley 26/1990 sobre pensiones no contributivas y las Leyes 22/1992 y 22/1993 de protección de desempleo. Esta legislación se complementará en la década de los noventa con nuevas normas que ordenarán el sistema de la Seguridad Social (Real Decreto Legislativo 1/1994, de 20 de junio, reiteradas veces modificado, entre otros por el Real Decreto-Ley 16/2001, de 27 de diciembre, de medidas para el establecimiento de un sistema de jubilación gradual y flexible), los intentos de conciliar la vida familiar y laboral de la mujer.

Pero este indudable desarrollo del modelo de bienestar presenta claroscuros cuando se constata los efectos producidos. Por una parte, como ya hemos comentado, los niveles de protección alcanzado nunca logran alcanzar los estándares europeos e incluso se sitúan en una posición divergente respecto de las prestaciones de los países 
comunitarios. Los numerosos estudios de Vicenç Navarro han puesto de relieve las limitaciones de las ayudas estatales, su parcialidad y su progresivo alejamiento de los niveles alcanzados en Europa. Por otra parte, sufren el efecto de los avatares sociales y económicos de estas décadas. La contención del gasto produce una baja cobertura y propicia la segmentación de la sociedad al distribuir de forma desigual los beneficios sociales.

La construcción del Estado Social español se ha realizado en unas condiciones históricas que han marcado su identidad. De la misma forma que ha estado condicionado el grado de desarrollo y cobertura y ha sufrido el impacto de la transformación de las condiciones económicas e ideológicas de la década de los noventa, también se han asumido las modificaciones que se han producido en la concepción de los derechos sociales. La denuncia sobre los excesos cometidos en la gestión de las prestaciones sociales subrayaba la situación de prepotencia administrativa y de postergación de los administrados que quedaban sometidos a la discrecionalidad burocrática y privados de la posibilidad de decidir por sí mismos. Habia, pues, una demanda de recuperación de la autonomía que no podía quedar desatendida.

Debemos, en principio, dejar constancia que la realización histórica del Estado es peculiar en cada país. A pesar de los intentos de predisponer modelos cerrados presididos por lógicas y desarrollos coherentes, la verificación empírica nos muestra la evolución de las bases comunes de acuerdo con las tradiciones políticas y culturales, con las capacidades económicas y con los conflictos y posiciones políticas. Todo ello conduce a la falta de correlación entre la forma de Estado constitucionalizada y su actuación legislativa, puesto que el resultado en cada país se dispersa en un amplio abanico de posibilidades en función de los sujetos beneficiarios, las necesidades atendidas, las formas jurídicas de protección y la distribución de las prestaciones. La ausencia de apriorismos o de lógicas inmanentes que nos permita deducir de los principios los desarrollos consiguientes, nos impone el análisis pormenorizado de los ordenamientos juridicos para detectar las novedades que se introducen.

Cuando se hace una enumeración de las técnicas jurídicas utilizadas para lograr la distribución de la riqueza se alude a las subvenciones, subsidios, acceso a los servicios públicos de forma gratuita 0 favorecido por precios políticos, al control de precios, a determinación de precios mínimos, a la reserva de bienes públicos, a la regulación de la producción, etc. En esta pléyade de posibilidades subyace la cró- 
nica tensión entre autoridad e individuo, entre la decisión discrecional y la autonomía del individuo, entre la prepotencia burocrática y la capacidad de decisión del ciudadano. La regulación jurídica de la política social permitía un uso arbitrario del poder que se traducía en la libre valoración de las necesidades y de los requisitos y en la posibilidad incontrolada de asignación de las ayudas, lo que contribuía a perpetuar la situación de inferioridad de los dependientes y a una asignación de ayudas no siempre coherente con las necesidades reales.

Afortunadamente se han introducido cambios en este modelo. Ya no se trata sólo de satisfacer necesidades sino de respetar la libertad y voluntad de los beneficiarios de las ayudas. La intervención administrativa, gestora de los recursos públicos aplicados a los colectivos necesitados, debe ser compatible con una capacidad de opción que permita una mayor adecuación a las condiciones de precariedad del ciudadano. Ya no hay un modelo rígido predeterminado sino que las ayudas se determinan adaptándose a las condiciones y cualidades de cada uno. Con este proceso de colaboración se intenta realizar un proceso basado en la participación, decisión y opción que permite la realización de un proyecto que, dentro de las difíciles condiciones de cada caso, se inspira en el dictado del artículo 10.1 CE sobre el libre desarrollo de la personalidad. De ahí que frente a la posición de la Administración y a la técnica del interés legítimo, se recurra al reconocimiento de los derechos subjetivos como garantía jurídica de acceso a las ayudas previstas en la política social. Esta subjetivización de las prestaciones, que responde al intento de traducir jurídicamente la relevancia alcanzada por el ideario individualista, la participación de los ciudadanos y la asunción de responsabilidades constituyen una de las principales manifestaciones de cambio, que afectan a los derechos sociales en esta época de transición.

\section{EL ESTADO SOCIAL AUTONÓMICO}

Una de las peculiaridades de nuestro modelo constitucional consiste en la distribución territorial del poder por medio del reconocimiento de autonomías políticas, dotadas de dirección política y de relevantes competencias para la gestión de sus intereses dentro de la unidad del orden constitucional. Esta división del poder ha generado históricamente la tensión entre centro y periferia, que en el caso que nos ocupa se traducía en la necesidad de disponer de un esquema de igualdad que impida la consolidación de diferencias entre los ciudadanos. El poder político territorial puede introducir, por razones polí- 
ticas o por disponibilidades económicas, un tratamiento jurídico que acentúe las desigualdades. Se genera de esta forma una doble dinámica: por una parte, evitar que las diferencias entre colectividades territoriales repercutan sobre la cohesión estatal; por otra parte, impedir que la defensa de la unidad desemboque en un uniformismo que ahogue la autonomía política territorial. A la primera cuestión se ha respondido con la aplicación del artículo 149.1.1 CE que atribuye al Estado, como competencia exclusiva, la regulación de las condiciones básicas que garanticen la igualdad de todos los españoles en el ejercicio de los derechos, precepto que no podemos comentar en esta ocasión; para el segundo tema, el Tribunal Constitucional admitió tempranamente la necesidad de distinguir entre unidad y uniformidad, la aceptación de una posición jurídica diversificada de los ciudadanos en las distintas partes del territorio nacional, si bien las Comunidades Autónomas no podían regular las condiciones básicas del ejercicio del derecho, y la constitucionalidad de la regulación autonómica sobre los derechos sociales en el ámbito de su competencia, dando así satisfacción a la reserva de ley prevista en la Carta Magna.

El reconocimiento de la autonomía política constitucional y la precisión de las competencias en los Estatutos ha permitido hablar, en consecuencia, de un Estado Social autonómico. El respeto a la competencia del artículo 149.1.1 es compatible con la capacidad de las Comunidades Autónomas de desarrollar una política social articulada y de establecer un régimen específico en la prestación de los derechos sociales.

Pero lo singular consiste no sólo en reconocer un espacio para la política autonómica, sino en considerar que el bloque de los derechos sociales es una competencia transferida y que corresponde a las Comunidades Autonómicas la relación primordial con los ciudadanos por medio de las prestaciones básicas, el entorno vital de los individuos (p.e. sanidad, educación, vivienda, enseñanza, servicios sociales). Que el Estado se reserve la competencia de la Seguridad Social responde a una distribución funcional de materias. Al afectar a la reproducción social, porque el conjunto de aportaciones de empresarios $y$ trabajadores incide en el precio de la mano de obra y en los costes de la producción, el Estado entiende en exclusiva de las condiciones de mercado. De esta forma se establece una división entre los poderes políticos: al Estado corresponde entender de las condiciones básicas de los ciudadanos y de los elementos que repercuten sobre el mercado y la reproducción social, mientras que a las Comunidades Autónomas se les confía el entorno vital de los ciudadanos. Este esquema está consagrado en la Constitución y en los Estatutos y dota a los poderes 
autonómicos de una capacidad de influir en las condiciones de vida y en el tejido social. El factor que corrige esta primera impresión consiste en la financiación. La negociación de las transferencias se realizó en condiciones desfavorables para las Comunidades Autónomas que están en una situación de dependencia de los fondos presupuestarios estatales.

En esta revisión de las prestaciones sociales es necesario reflejar los cambios que se han producido y las repercusiones inducidas por la acelerada transformación sufrida en las condiciones económicas de nuestras sociedades. El esquema de reproducción se basaba en un mercado que permitía a un trabajador con un empleo estable disponer de los recursos con los que atender a las necesidades de su familia, la cual recibia, por otra parte, las prestaciones públicas que aseguraban el consumo social necesario para el buen funcionamiento del sistema. Este modelo fundado en el pleno empleo y en el trabajador masculino y padre de familia, quiebra con la transformación económica, social y política de los años 70, que se acentúa en los años 80 y produce un cambio radical en el espacio político. Estas breves consideraciones nos sirven de introducción para referirnos al problema de las repercusiones que sobre las prestaciones sociales produce el nuevo escenario, tan distante de las previsiones constitucionales.

A pesar de la ofensiva ideológica lanzada contra el Estado Social y los esfuerzos políticos desplegados en pro de su desmantelamiento, se produce un cuestionamiento de la igualdad sustancial, la desaparición de la intervención directa en la economía por medio de las privatizaciones, la liberalización de la actividad económica, la transformación de las prestaciones sociales. Aunque no se logra la desaparición del modelo social, se establece una diversificación de la acción social: a) se mantienen las prestaciones en los derechos de legitimación (enseñanza, sanidad, etc.) imprescindibles para el funcionamiento del sistema; b) se incide drásticamente en el área del trabajo por medio de una profunda revisión del mercado laboral que disminuye el garantismo de la regulación jurídica, y empeora el régimen de la Seguridad Social; c) se atiende a las nuevas necesidades surgidas como consecuencia del desempleo, los cambios demográficos, la inmigración. Será en este último ámbito en el que se produzca la acción autonómica más novedosa.

En las nuevas condiciones los servicios sociales, pensados como complemento secundario del funcionamiento del mercado, adquieren un gran protagonismo como instrumento para atender los problemas generados por la marginalidad y pobreza. Desde 1982, después de la 
legislación pionera del País Vasco, todas las Comunidades Autónomas introducen unas prestaciones que las legitiman y que afrontan fenómenos lesivos para la cohesión social. De nuevo con brevedad, subrayamos los aspectos más significativos.

Sobre las Comunidades Autónomas han gravitado simultáneamente la emergencia de nuevas necesidades y las condiciones de crisis del Estado Social en las que tiene que producirse la respuesta. La nueva acción social se realizará en un contexto transformado: los problemas surgidos, la revisión de la función de la Administración y la contención del gasto social, la relación sociedad-Administración y la búsqueda de la implicación del individuo en las alternativas a su situación. Para todas estas cuestiones se aprestará una respuesta. Por una parte, la legislación de los servicios sociales afrontará los problemas de pobreza y marginalidad. Las sucesivas reformas se han aproximado a la configuración de un derecho subjetivo e intentado superar las limitaciones presupuestarias que limitaban el alcance de las prestaciones. En segundo lugar, se ha procedido a regular la figura del voluntariado que simultáneamente es una vía de solidaridad y de implicación social, de sustitución de la Administración, de mercantilización de la acción social y de reducción de los costes. Por último, frente al desempleo y la marginalidad, para evitar la perpetuación de la situación de exclusión, se ha arbitrado la fórmula del salario social que pretende establecer un acuerdo entre la Administración y el individuo para superar su situación de dependencia. También en este campo se ha evolucionado hacia las formas de subjetivización de las prestaciones y hacia el impulso de la opción y participación de los beneficiarios. Dos consecuencias se derivan de esta propuesta: en primer lugar, los análisis empíricos realizados confirman el escaso éxito redentor de estas fórmulas; en segundo lugar, se ha abierto el debate sobre la renta básica como vía para la superación de las carencias de las políticas realizadas.

En todo caso, estamos ante una cuestión sensible y dinámica, que sufre los embates de las cambiantes circunstancias políticas e institucionales. Inmersos como estamos en procesos de cambios de diversa índole, son múltiples aspectos que quedan afectados referidos a los sujetos, necesidades, prestaciones y requisitos. Así, por mencionar sólo dos aspectos, podemos recordar el indudable efecto de la estabilidad presupuestaria promovida en la Unión Europea e interiorizada en nuestro ordenamiento por medio de la Ley Orgánica 5/2001 y la Ley 18/2001, lo que supone, contra la lógica del Estado Social, establecer un límite de gasto que no puede no repercutir sobre los derechos sociales. Por otra parte, es permanente la conflictividad entre el Estado y 
las Comunidades. En sus relaciones verificamos una continua pugna que presenta variedad de vicisitudes según las materias. Así, por una parte, se emprende un proceso de racionalización en el ámbito de sanidad con una normativa consensuada, mientras que se mantiene la divergencia en la materia de las pensiones no contributivas con posiciones encontradas entre el Estado y las Comunidades Autónomas.

\section{CONCLUSIONES}

A pesar de la crisis del Estado Social como forma de organización política, los derechos sociales conservan su vigencia. La ofensiva de los ideólogos del neoliberalismo contra las prestaciones públicas no consiguió traspasar la esfera doctrinal. Durante la gestión de los gobiernos conservadores no se consiguió desmantelar el patrimonio jurídico, institucional y económico acumulado durante años de inspiración socialdemócrata. Los intentos de reducción o eliminación han topado siempre con una opinión pública que en el espacio comunitario ha mostrado su adhesión hacia el sistema de bienestar e incluso en algunos países desarrollado una dura resistencia contra los intentos gubernamentales de revisión del régimen jurídico prestacional. Esta buena salud encontraría una ulterior confirmación en su recepción en el ámbito comunitario. Con independencia de cuál sea su naturaleza y de su compatibilidad con la juridificación de un modelo de economía social de mercado altamente competitiva, la Carta de Derechos Fundamentales ha articulado el modelo social europeo con la mención expresa a diversos derechos sociales.

Pero la supervivencia nominal de los derechos sociales no debe eximirnos de valorar las modificaciones que se han producido. Como es habitual en estos temas, hay que referirse a la convivencia de aspectos contradictorios. Si los derechos sociales han servido simultáneamente para mejorar las condiciones de vida de los ciudadanos y para el funcionamiento de la economia, en la actualidad se produce una adaptación a las condiciones que dimanan de la globalización.

Aunque se ha producido una universalización de derechos sociales, aunque se han creado nuevo derechos, aunque se ha fomentado la libertad y opción de los beneficiarios, aunque se ha favorecido la participación social, aunque se ha acercado la gestión y el control a los administrados, no se pueden desconocer las novedades contradictorias que se han producido. La desaparición del pleno empleo, las bolsas de desempleo y la llegada de inmigrantes han erosionado la base 
sobre la que se asentaban los derechos sociales: el trabajo. Es cierto que los cambios producidos durante el último tercio de siglo han fragmentado la clase trabajadora y la sociedad y han transformado el fundamento clasista de las prestaciones por una base de tipo democrática. Pero continúa vigente que en una economia capitalista, mientras no se impongan las propuestas de la renta básica universal, el eje de la satisfacción de necesidades pasa por el mercado y los derechos sociales se materializan precisamente en quienes disfrutan de una posición en el mercado laboral. Cuando en la globalización y sus secuelas se consolidan formas de desempleo y, sobre todo, se instauran la flexibilidad y la precariedad laboral, la atribución jurídica de los derechos sociales no es capaz de paliar los efectos nocivos de la nueva estructura social y económica. El problema básico consiste en que se frustra el fundamento que antes reconociamos a los derechos sociales. Como es comúnmente admitido, el desafío ya no es avanzar en la implantación de una igualdad sustancial sino en evitar la fragmentación social y en la creación de espacios de marginalidad. La necesidad de adaptación a las exigencias de la globalización conduce a una contención del gasto, a un endurecimiento de los requisitos de accesos, a una reducción de las prestaciones, a las fórmulas de copago, a la irrupción de la iniciativa privada en la esfera de bienestar, al desplazamiento de la responsabilidad pública por la intervención social, a la sustitución del Estado por los poderes regionales y locales en una división del trabajo que encomienda al primero la adaptación a la economía y a los segundos las tareas asistenciales, que denotan al deterioro del mercado de trabajo y la renuncia a la consecución de la igualdad sustancial. Aunque se mantiene a la baja el gasto público, el objetivo es el de sostener los mínimos vitales y el resultado es la fragmentación social. Se ha dado importantes cambios en la provisión de prestaciones (ya sea pública o privada), en la organización (centralizada o descentralizada), en los contenidos (de la igualdad sustancial al criterio asistencial, de la ampliación al endurecimiento de los requisitos), en los sujetos (dirigidos al conjunto de los ciudadanos o los excluidos), en la naturaleza jurídica ( de los intereses a los derechos subjetivos), en el disfrute (del paternalismo administrativo a la responsabilidad individual). Pero el balance que se extrae de todas estas modificaciones es que, por encima de la preservación del texto constitucional, cambia el significado de los derechos sociales inmersos en una lógica distinta a la de su inspiración que consagra un nuevo fundamento y un diverso objetivo: la primacía de la asistencia frente al imperativo constitucional de la igualdad sustancial. 